\title{
Overexpression of MT1-MMP is insufficient to increase experimental liver metastasis of human colon cancer cells
}

\author{
HIROFUMI YAMAMOTO ${ }^{1}$, SHINGO NOURA ${ }^{2}$, JIRO OKAMI $^{2}$, MAMORU UEMURA $^{1}$, \\ ICHIRO TAKEMASA ${ }^{1}$, MASATAKA IKEDA ${ }^{1}$, HIDESHI ISHII ${ }^{1}$, MITSUGU SEKIMOTO ${ }^{1}$, \\ NARIAKI MATSUURA ${ }^{3}$, MORITO MONDEN ${ }^{1}$ and MASAKI MORI ${ }^{1}$ \\ ${ }^{1}$ Department of Surgery, Gastroenterological Surgery, Graduate School of Medicine, Osaka University; \\ ${ }^{2}$ Department of Surgery, Osaka Medical Center for Cancer and Cardiovascular Diseases; ${ }^{3}$ Department of \\ Pathology, School of Allied Health Science, Faculty of Medicine, Osaka University, Osaka, Japan
}

Received June 13, 2008; Accepted August 1, 2008

DOI: 10.3892/ijmm_00000082

\begin{abstract}
The expression and activation of matrix metalloproteinases (MMPs) by tumor cells is correlated with invasive and metastatic potential. The purpose of this study was to examine the impact of increased membrane type 1 matrix metalloproteinase (MT1-MMP) expression on liver metastatic potential utilizing human colorectal cancer (CRC) cell lines. Three human CRC cell lines, DLD1, HCT116 and HT29, were stably transfected with the MT1-MMP cDNA, and experimental liver metastasis was established by injecting the cells into the spleens of nude mice. Reverse transcriptionpolymerase chain reaction (RT-PCR) confirmed increased expression of MT1-MMP mRNA in the stable tranfectants. In vitro analysis by gelatin zymography and morphological survey demonstrated that MT1-MMP transfectants displayed a matured gelatinolytic activity and invasive properties when cultured in 3D collagen gel, indicating that transduced MT1MMP cDNA was functional. Although there was no difference in cell proliferation rate between MT1-MMP overexpressing cells and the Mock control cells, in vivo experiments indicated that the liver metastatic ability was not affected by MT1MMP overexpression. Our findings indicated that conditional MT1-MMP overexpression was insufficient to increase
\end{abstract}

Correspondence to: Dr Hirofumi Yamamoto, Department of Surgery, Gastroenterological Surgery, Graduate School of Medicine, Osaka University, 2-2 Yamada-oka, Suita-City, Osaka 565-0871, Japan

E-mail: kobunyam@surg2.med.osaka-u.ac.jp

Abbreviations: CRC, colorectal cancer; ECM, extracellular matrix; FBS, fetal bovine serum; MT-1 MMP, membrane type 1 matrix metalloproteinase; RT, reverse transcription; PBGD, phorphobilinogen deaminase; PCR, polymerase chain reaction; TIMP, tissue inhibitors of matrix metalloproteinase

Key words: MT1-MMP, colorectal cancer, invasion, liver metastasis experimental liver metastasis, suggesting a more complicated mechanism may be involved in the activation and regulation of MMPs cascades in vivo.

\section{Introduction}

Destruction of the extracellular matrix (ECM), especially the basement membrane, is an essential step in tumor invasion and metastasis. Degradation of the ECM generates a new avenue for invasion and allows movement of tumor cells across basement membrane. A major family of ECM degrading enzymes involved in this process is the matrix metalloproteinases (MMPs) family (1). MMPs are generally expressed at low level and induced in events that required ECM remodeling such as wound healing. Most MMPs are secreted in a latent form that requires proteolytic removal of the $\mathrm{N}$-terminal propeptide to become catalytically active (2). These proteinases are regulated at transcription level and also at translation level and activated through induction of proteolytic cascades (3). Membrane-bound membrane type 1 matrix metalloproteinase (MT1-MMP) activates pro-MMP-2, which is shown to contribute to the invasive abilities of various tumors (4).

MT1-MMP, also termed MMP-14, is a transmembrane protein expressed in an active form (5). It cleaves or degrades various kinds of the ECM compounds including collagen, gelatin and fibrin and facilitates invasion and metastasis of tumor cells. MT1-MMP also activates several downstream MMPs, including the most widespread gelatinase, MMP-2 (gelatinase A) (6). There is evidence that mechanisms as diverse as autocatalytic processing, ectodomain shedding, homodimerization and internalization can all contribute to the modulation of MT1-MMP activity on the cell surface (3). The dynamic turnover of MT1-MMP at the migration edge by internalization is important for proper enzyme function during cell migration and invasion (7). MT1-MMP expression has been documented in several types of tumors and upregulation of MT1-MMP is strongly implicated in tumor growth and metastasis spread (8-10). In colorectal cancer (CRC), MT1-MMP overexpression is noted during multistage tumorigenesis (11), and tumor phenotype stratified by 
Table 1. PCR primers.

\begin{tabular}{|c|c|c|c|}
\hline Gene & Sense primer & Antisense primer & Product size (bp) \\
\hline MT1-MMP & 5'-ACCTACGTACCCACACACAG-3' & 5'-AAATTCTCCGTGTCCATCCA-3' & 661 \\
\hline PBGD & 5'-AACGGCGGAAGAAAACAG-3' & 5'-TCCAATCTTAGAGAGTGCA-3' & 190 \\
\hline
\end{tabular}

both MMP and tissue inhibitors of matrix metalloproteinase (TIMP) is associated with poor prognosis (12).

Since liver metastasis is one of the most frequent causes of death from CRC (13), we investigated whether MT1MMP overexpression would contribute to facilitate formation of liver metastasis using 3 types of CRC cell lines stably tranfected with MT1-MMP cDNA. To our knowledge, this is the first study that investigated effects of MT1-MMP on liver metastatic potential using in vivo experimental liver metastasis model.

\section{Materials and methods}

Cell lines. Three human CRC cell lines, namely DLD1, HCT119 and HT29 were obtained from the American Type Culture Collection (Manassas, VA). These cell were maintained in Dulbecco's modified Eagle's medium (DMEM) supplemented with $10 \%$ fetal bovine serum (FBS), $100 \mathrm{U} / \mathrm{ml}$ penicillin, and $100 \mu \mathrm{g} / \mathrm{ml}$ streptomycin in $5 \% \mathrm{CO}_{2}$ at $37^{\circ} \mathrm{C}$.

Expression plasmid and transfection. MT1-MMP expression plasmid with neomycin resistance was kindly provided by Professor S. Weiss from University of Michigan (14,15). A pcDNA3 vector (Invitrogen, Carlsbad, CA) was used as the control vector. Cell transfection was performed, as described previously (16). Stable transfectants were selected for 10-14 days using G418 (Sigma, Ronkonkoma, NY) at concentration of $0.9 \mathrm{mg} / \mathrm{ml}$ in complete medium. Each plate containing hundreds of resistant clones was trypsinized and 10-20 colonies were picked up on 96-well plates, maintained in complete DMEM medium containing $0.5 \mathrm{mg} / \mathrm{ml} \mathrm{G} 418$. After cell expansion, each clone was subject to RT-PCR assay for MT1-MMP expression and the 2 clones that stably expressed the highest level of MT1-MMP mRNA, were used for subsequent analysis. As experimental controls, a pool mixture and a single control clone were used.

Reverse transtriptase-polymerase chain reaction (RT-PCR). Total RNA was extracted with Trizol reagent (Life Technologies, Gaithersburg, MD). Complementary DNA (cDNA) was generated using myeloblastosis virus reverse transcriptase (Promega, Madison, WI). Reverse transcription (RT) was performed at $42^{\circ} \mathrm{C}$ for $90 \mathrm{~min}$, followed by heating at $95^{\circ} \mathrm{C}$ for $5 \mathrm{~min}(17)$. A housekeeping gene, phorphobilinogen deaminase (PBGD) was used as an internal control (18). The PCR primers for MT1-MMP, and PBGD are shown in Table I. PCR conditions were: initial denaturing at $95^{\circ} \mathrm{C}$ for $12 \mathrm{~min}$, followed by $35-40$ cycles of $95^{\circ} \mathrm{C}$ for $1 \mathrm{~min}, 62^{\circ} \mathrm{C}$ for $1 \mathrm{~min}$ and $72^{\circ} \mathrm{C}$ for $1 \mathrm{~min}$, and a final extension at $72^{\circ} \mathrm{C}$ for $10 \mathrm{~min}$. PCR products $(661 \mathrm{bp})$ were electrophoresed on a $2 \%$ agarose

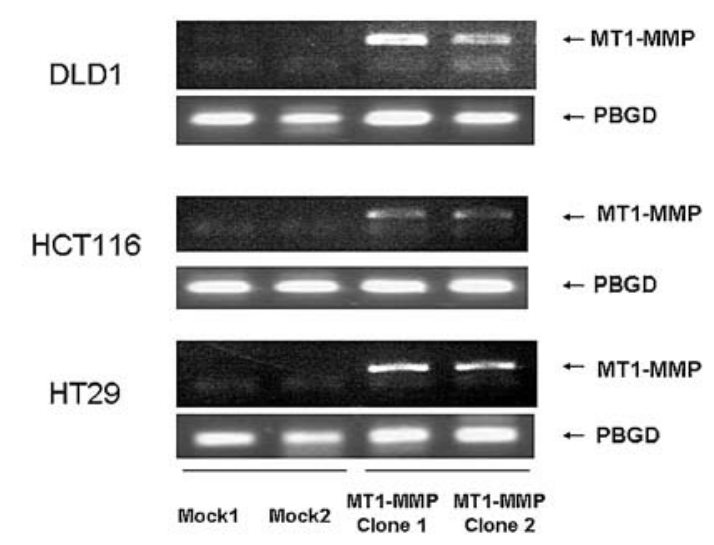

Figure 1. MT1-MMP mRNA expression. MT1-MMP mRNA expression was assessed by RT-PCR. High level of MT1-MMP mRNA was commonly noted in the stable transfectants of MT1-MMP cDNA, although endogenous MT1-MMP mRNA was scarce in three CRC cell lines. Two MT1-MMP expressing clones derived from single colony (Clone1, Clone 2) and two Mock control cultures (Mock1, pool; Mock2, single colony) are shown for each cell type. PBGD served as internal control.

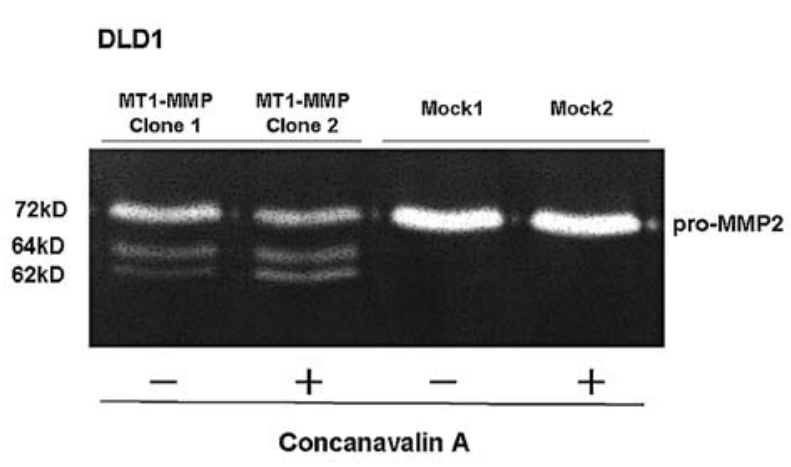

Figure 2. MT1-MMP overexpression induced gelatinolytic activity. Representative results of gelatinolytic activity in MT1-MMP expressing DLD1 cells. Both Mock controls and MT1-MMP expressing clones were treated with or without concanavalin A (Con A), which is known to enhance the cell ability to activate pro-MMP2 (19). Conditioned media were analyzed by zymography. Pro, intermediate and active forms are as indicated at 72,64 and $62 \mathrm{kDa}$.

gel, stained with ethidium bromide and visualized under UV transillumination.

Gelatin zymography. Zymography was performed to assess the gelatinolytic activity of MT1-MMP, as previously described (19). Briefly, $24 \mathrm{~h}$ after seeding, cell were incubated with serum-free medium with or without $5 \mu \mathrm{M}$ concanavalin A (Sigma) for another $48 \mathrm{~h}$. Samples of conditioned medium were collected and electrophoresed on a polyacrylamide gel containing $0.1 \%$ gelatin. Substrate digestion were performed 


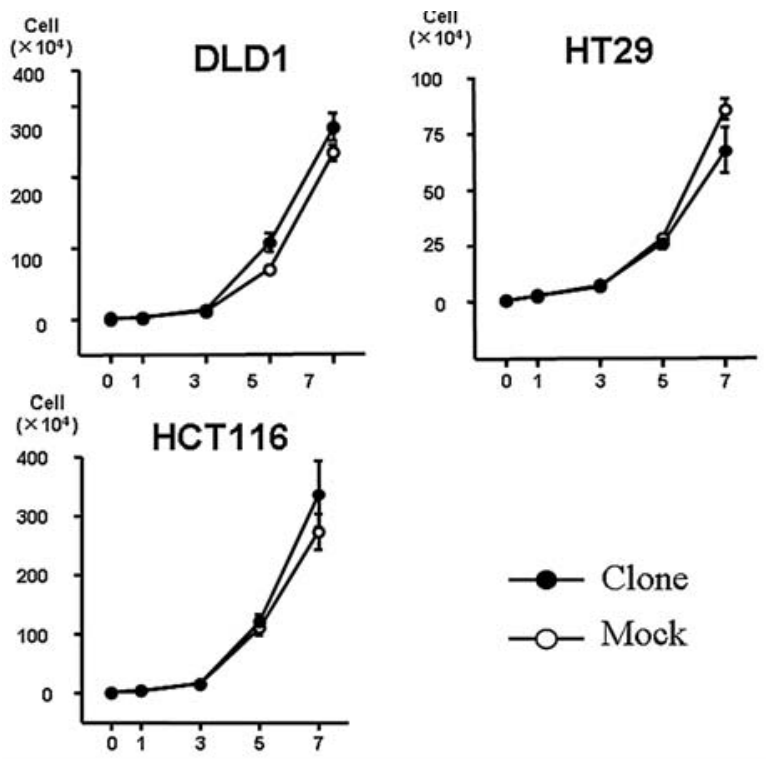

Figure 3. Cell proliferation assay. Cell counts were performed on Day 1, 3, 5 and 7. No statistically significant difference was found between the Mock control (Mock) or MT1-MMP expressing clones (Clone). Results are expressed as the mean \pm SD. A repeat experiment showed similar results (data not shown).

by incubating gel in $50 \mathrm{mM}$ Tris- $\mathrm{HCl}(\mathrm{pH} 7.5)$ containing $5 \mathrm{mM} \mathrm{CaCl}{ }_{2}, 1 \mu \mathrm{M} \mathrm{ZnCl}_{2}$ at $37^{\circ} \mathrm{C}$ overnight with gentle rocking.

Cell growth. Cells were seeded at $1 \times 10^{4}$ in 6-well plate in triplicate and cultured in a complete medium containing $10 \%$ FBS. Cell count was performed on day 1, 3, 5 and 7, respectively. Data were expressed as mean \pm standard deviation (SD).

Three-dimentional cell culture. Cells were cultured in triplicate in collagen gel (Nitta Gelatin, Osaka, Japan) in 12-well plate according to manucfacturer's instructions. Collagen $(1 \mathrm{ml})$ containing $5 \times 10^{5}$ cells was polymerized in each well, followed by overlaying $1 \mathrm{ml}$ DMEM. Culture medium was changed every $24 \mathrm{~h}$. On the 10 th day, the colonies $>50 \mu \mathrm{m}$ were counted in 5 fields at high magnification. Data are expressed as mean \pm SD.

Experimental liver metastasis model. Specific pathogen-free 4-week-old BALB/c-nu/nu nude mice were used for in vivo experiments (Clea Japan, Inc., Osaka, Japan). Viable cancer cells $\left(5 \times 10^{6}\right.$ cell in $50 \mu 1$ of cell suspension) were injected into the spleen of nude mice under light ether anesthesia, then spleen was removed after vessel ligation. Six weeks later, mice were sacrificed to determine the incidence of liver metastasis. All of the animal experiments were performed in accordance with the Guidance for the Care and Use of Laboratory Animals in Osaka University.

Statistical analysis. Statistical analysis was performed using the StatView J-5.0 program (Abacus Concepts, CA). Data were analyzed using Student's t-test and considered to be significant at $\mathrm{p}<0.05$.
Table II. Incidence of liver metastasis.

\begin{tabular}{|c|c|c|c|c|c|c|}
\hline \multirow{2}{*}{$\begin{array}{l}\text { Number } \\
\text { of foci }\end{array}$} & \multicolumn{2}{|c|}{ DLD1 } & \multicolumn{2}{|c|}{ HCT116 } & \multicolumn{2}{|c|}{ HT29 } \\
\hline & Clone & Mock & Clone & Mock & Clone & Mock \\
\hline 0 & 5 & 8 & 0 & 1 & 2 & 1 \\
\hline $1-10^{a}$ & 0 & 3 & 3 & 3 & 7 & 8 \\
\hline Total & 5 & 11 & 3 & 4 & 9 & 9 \\
\hline
\end{tabular}

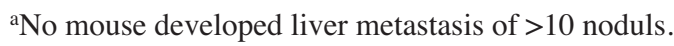

\section{Results}

Establishment of MT1-MMP stable transfectant. Expression of MT1-MMP mRNA in the CRC cell lines, DLD1, HCT116 and HT29 transfected with either control vector (Mock) or MT1-MMP expression plasmid (Clone), is shown in Fig. 1. Endogenous expression of MT1-MMP mRNA was scarce in 3 cell lines, while all stable tranfectants of MT1-MMP cDNA displayed an increased expression of MT1-MMP mRNA.

Gelatinolytic activity by MT1-MMP overexpression. The ability of MT1-MMP to activate MMP-2 was then examined using zymography. Fig. 2 showed that DLD 1 clone was able to convert pro-MMP2 $(72 \mathrm{kD})$ to an active form $(62 \mathrm{kD})$ and an intermediate form $(64 \mathrm{kD})$. Treatment with con-canavalin A accelerated the activation process. On the other hand, the pro-MMP remained uncleaved in the Mock control cells even in the presence of concanavalin A. Similar results were obtained in two other CRC cell lines (data not shown).

Cell proliferation assay. In three CRC cell lines, no significant difference was noted in cell proliferation between MT1-MMP tranfectants and the Mock control cells (Fig. 3). A repeat experiment showed similar results (data not shown).

$3 D$ cell invasion assay. In the collagen gel, apparently invasive cell feature displaying irregular shape was observed in MT1MMP tranfectants compared to the Mock control cells which uniformely formed small and round colonies (Fig. 4A). When the colonies with a diameter $>50 \mu \mathrm{M}$ were counted, a larger population was observed in MT1-MMP transfectants compared to the Mock control cells (Fig. 4B). Statistical significance was noted in all three cell lines.

Effects of MT1-MMP expression on experimental liver metastasis. We further examined the ability of liver metastatic induction between the MT1-MMP tranfectants and the Mock control cells when they were injected into the spleen. Table II showed the number of metastatic foci per mouse and the number of mouse developing liver metastasis. The size of metastatic foci was exclusively $<5 \mathrm{~mm}$ in $3 \mathrm{CRC}$ cells (data not shown). The results showed MT1-MMP expression did not affect the potential of liver metastasis. In DLD1 cells, the control cells established liver metastasis in 3 of 11 mice, but MT1-MMP transfectants did not produce hepatic metastasis 
A

\section{DLD1}

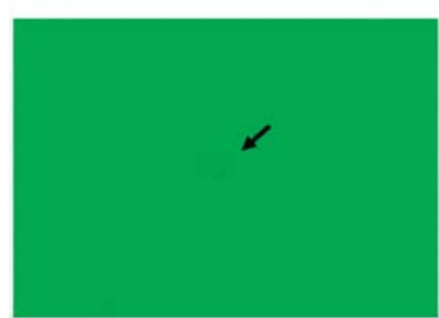

Mock

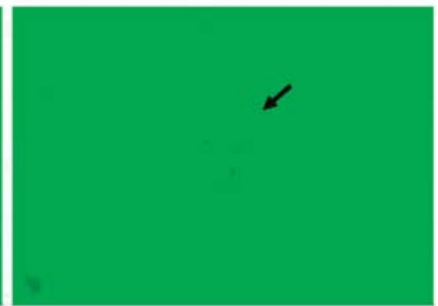

MT1-MMP Clone
B

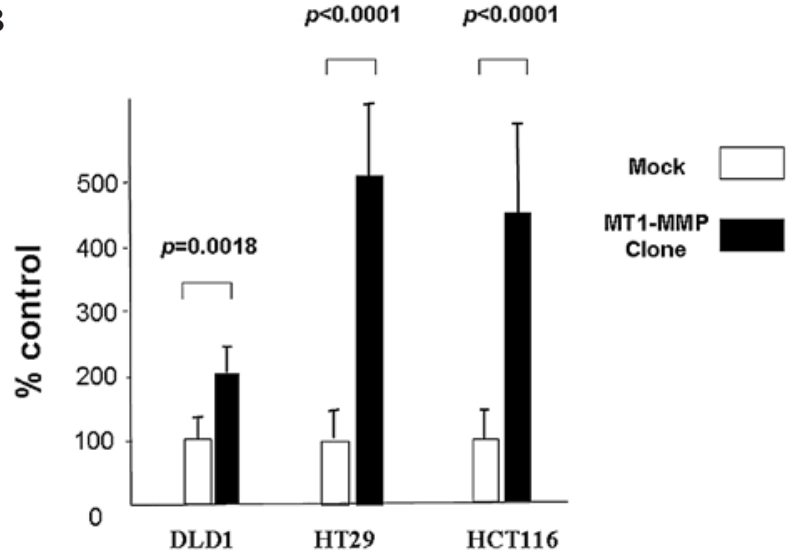

Figure 4. Cell invasion assay. (A) DLD cells cultured in collagen gel. The Mock control cells (Mock) were in tiny spherical form whereas the MT1MMP expressing cells (Clone) were often larger and showed an expanding irregular form. These representative cells are indicated by arrows. (B) Cell count on the 10th day indicated cells $>50 \mu \mathrm{m}$ were more commonly found in MT1-MMP-expressing cells compared to Mock control cells. In each cell line, the value of Mock control was adjusted as $100 \%$. P-value indicated statistical significance in three CRC cell lines.

at all. In HCT116 and HT29 cells, liver metastases were relatively readily established, but quite similar number and size of metastatic foci were observed between MT1-MMP clones and Mock controls.

\section{Discussion}

MT1-MMP is implicated in the tumor invasion and metastasis process since it activates MMP-2 that degrades a major component of the basement membrane, the type IV collagen. Previous studies reported that conditional MT1-MMP overexpression contributed to enhanced potential of lymph node and lung metastasis of prostate cancer cells (20) and lymph node metastasis of gastric cancer cells (21) by orthotopic implantation model. Forced expression of MT1-MMP in tumor cells was also shown to enhance pulmonary metastases in an experimental metastasis assay (22). Liver metastasis is a frequent cause of death from CRC (13), but no study has been conducted so far to investigate whether the increased expression of MT1-MMP in CRC cells would be sufficient to induce experimental liver metastasis in vivo.
In vitro analysis of cell proliferation did not show any obvious differences between MT1-MMP overexpressing clones and the Mock control cells, indicating that expression of MT1-MMP does not stimulate proliferation of CRC cells. To date, the consensus that proteinases do not regulate tumor cell growth directly has been achieved on in vitro model systems wherein cancer cell behavior is classically assessed in a monolayer environment. However, it is worth noting that an increase in cell number in MT1-MMP overexpressing cells has been documented under 3D culture conditions $(23,24)$. Putatively, when cancer cells are forced to proliferate within a dense $3 \mathrm{D}$ matrix, the invading capacity is a key factor that influences the tumor growth. Besides enhancement of growth of CRC cells, our results of 3D collagen cultures showed that forced expression of MT1-MMP enhanced the gelatinolytic activity, and induced invasive potential, being consistent with previous studies $(25,26)$. These findings indicate that transduced MT1-MMP cDNA is functional in 3 CRC cell lines produced in this study.

On the contrary, increased MT1-MMP expression alone had no effect on the liver metastatic potential, when cells were injected into spleen, thus positioned directly into the blood flow. Tumor progression is a complex multistep process involving a series of different biological obstacles that a tumor cell must overcome to establish metastasis (27). Firstly, the tumor cell must invade locally, then intravasate into the circulation. Degradation of the ECM component by MMPs to remove the physical barrier is indeed the initial stage of metastasis. Soulie et al found that expression of MT1-MMP in non-tumorigenic, well-differentiated epithelial cells was sufficient to activate cellular responses involved in tumor initiation, local tissue invasion and intravasation (23). In the subsequent steps, at the same time, tumor cells have to execute various oncogenic properties that are required for survival in the circulation, adhesion to the endothelium, extravasation from the vessels, cell proliferation, and neovascularization. In this context, one may suppose that orthotopic implantation into the colonic wall will provide more evident effects by MT1-MMP, as reported by others $(20,21)$. However, the scenario may not be so simple since there is evidence that forced expression of MT1-MMP in mouse carcinoma cells significantly increased number of lung nodules even in experimental metastasis assay, i.e., by injection of the cells into mouse tail vein (22). This suggests that MT1-MMP could have a role also during extravasation and/or in forming lung metastatic lesion.

It is known that the tumor-stroma interaction, especially pro-MMP2 produced by stromal cell (most probably by fibroblasts), is important for establishment of liver metastasis $(3,6,12)$. When pro-MMP2 is activated by MT1-MMP, TIMP-2 is particular important to modulate the activity negatively (3). An imbalance between the proteolytic activity of MMP-2 and TIMP-2 is shown to be clinically important in hepatocellar carcinoma, gall bladder carcinoma, and hilar cholangiocarcinoma (28-30). Moreover, Brand et al previously reported that adenoviral transfer of TIMP-2 into the liver tissue suppressed liver metastasis (31). Therefore, another possibility might be that the conditional MT1-MMP overexpression in vivo could be counteracted by TIMP-2 derived from liver tissue. 
Undoubtedly, MT1-MMP has a role in tumor invasion and metastasis. However, our data showed that MT1-MMP expression led to the development of invasive properties of CRC cells but these characteristics were insufficient to promote the formation of liver metastasis in mouse experimental model. Our findings suggest that a more complicated mechanism may be involved in the activation and regulation of MMPs cascades in vivo metastasis to liver.

\section{Acknowledgements}

We are grateful to Nobuaki Hiraoka for valuable technical advice in gelatinolytic activity assay and invasion assay in collagen.

\section{References}

1. Hornebeck W, Emonard H, Monboisse JC and Bellon G: Matrix-directed regulation of pericellular proteolysis and tumor progression. Semin Cancer Biol 12: 231-241, 2002.

2. Nagase H: Activation mechanisms of matrix metalloproteinases. Biol Chem 378: 151-160, 1997.

3. Osenkowski P, Toth M and Fridman R: Processing, shedding, and ecdocytosis of membrane type 1-matrix metalloproteinase (MT1-MMP). J Cell Physiol 200: 2-10, 2004.

4. Curran S and Murray GI: Matrix metalloproteinases: molecular aspects of their roles in tumour invasion and metastasis. Eur $\mathbf{J}$ Cancer 36: 1621-1630, 2000.

5. Sato H, Takino T, Okada Y, Cao J, Shinagawa A, Yamamoto E and Seiki M: A matrix metalloproteinase expressed on the surface of invasive tumour cells. Nature 370: 61-65, 1994.

6. Maquoi E, Frankenne F, Baramova E, et al: Membrane type 1 matrix metalloproteinase-associated degradation of tissue inhibitor of metalloproteinase 2 in human tumor cell lines. J Biol Chem 275: 11368-11378, 2000.

7. Uekita T, Itoh Y, Yana I, Ohno H and Seiki M: Cytoplasmic tail-dependent internalization of membrane-type 1 matrix metalloproteinase is important for its invasion-promoting activity. J Cell Biol 155: 1345-1356, 2001.

8. Kikuchi R, Noguchi T, Takeno S, Kubo N and Uchida Y: Immunohistochemical detection of membrane-type-1-matrix metalloproteinase in colorectal carcinoma. Br J Cancer 83: 215-218, 2000.

9. Deryugina EI, Soroceanu L and Strongin AY: Up-regulation of vascular endothelial growth factor by membrane type 1 matrix metalloproteinase stimulates human glioma xenograft growth and angiogenesis. Cancer Res 62: 580-588, 2002.

10. Seiki M: Membrane-type 1 matrix metalloproteinase: a key enzyme for tumor invasion. Cancer Lett 194: 1-11, 2003.

11. Malhotra S, Newman E, Eisenberg D, Scholes J, Wieczorek R, Mignatti $P$ and Shamamian P: Increased membrane type a matrix metalloproteinase expression from adenoma to colon cancer: a possible mechanism of neoplastic progression. Dis Colon Rectum 45: 537-543, 2002.

12. Curran S, Dundas SR, Buxton J, Leeman MF, Ramsay R and Murray GI: Matrix metalloproteinase/tissue inhibitors of matrix metalloproteinase phenotype identifies poor prognosis colorectal cancers. Clin Cancer Res 10: 8229-8234, 2004.

13. Kobayashi H, Mochizuki H, Sugihara K, et al: Characteristics of recurrence and surveillance tools after curative resection for colorectal cancer: a multicenter study. Surgery 141: 67-75, 2007.
14. Pei D and Weiss SJ: Transmembrane-deletion mutants of the membrane-type matrix metalloproteinase-1 process progelatinase A and express intrinsic matrix-degrading activity. J Biol Chem 271: 9135-9140, 1996.

15. Yana I and Weiss S: Regulation of membrane type-1 matrix metalloproteinase activation by proprotein convertases. Mol Biol Cell 11: 2387-2401, 2000.

16. Deryugina EI, Bourdon MA, Luo GX, Reisfeld RA and Strongin A: Matrix metalloproteinase-2 activation modulates glioma cell migration. J Cell Sci 110: 2473-2482, 1997.

17. Ogawa M, Yamamoto $\mathrm{H}$, Nagano $\mathrm{H}$, et al: Hepatic expression of ANG2 RNA in metastatic colorectal cancer. Hepatology 39: 528-539, 2004.

18. Chretien S, Dubart A, Beaupain D, et al: Alternative transcription and splicing of the human porphobilinogen deaminase gene result either in tissue-specific or in housekeeping expression. Proc Natl Acad Sci USA 85: 6-10, 1988

19. Okami J, Nakamori S, Hiraoka N, et al: Suppression of pancreatic cancer cell invasion by a cyclooxygenase-2-specific inhibitor. Clin Exp Metastasis 20: 577-584, 2003.

20. Cao J, Chiarelli C, Kozarekar P and Adler HL: Membrane type1-matrix metalloproteinase promotes human prostate cancer invasion. Thromb Haemost 93: 770-778, 2005.

21. Yonemura Y, Endo Y, Takino T, et al: Membrane-type 1 matrix metalloproteinase enhances lymph nodes metastasis of gastric cancer. Clin Exp Metastasis 18: 321-327, 2000.

22. Tsunezuka Y, Kinoh H, Takino T, et al: Expression of membranetype matrix metalloproteinase (MT1-MMP) in tumor cells enhances pulmonary metastases in an experimental metastasis assay. Cancer Res 56: 5678-5683, 1996.

23. Soulie P, Carrozzino F, Pepper MS, Strongin AY, Poupon MF and Montesano R: Membrane-type-1 matrix metalloproteinase confers tumorigenicity on nonmalignant epithelial cells. Oncogene 24: 1689-1697, 2005.

24. Hotary KB, Allen ED, Brooks PC, Datta NS, Long MW and Weiss SJ: Membrane type I matrix metalloproteinase usurps tumor growth control imposed by the three-dimensional extracellular matrix. Cell 114: 33-45, 2003.

25. Wang P, Nie J and Pei D: The hemopexin domain of membranetype matrix metalloproteinase-1 (MT1-MMP) is not required for its activation of proMMP2 on cell surface but is essential for MT1-MMP-mediated invasion in three-dimensional type I collagen. J Biol Chem 279: 51148-51155, 2004

26. Bartolome RA, Galvez BG, Longo N, et al: Stromal cellderived factor-1alpha promotes melanoma cell invasion across basement membranes involving stimulation of membrane-type 1 matrix metalloproteinase and Rho GTPase activities. Cancer Res 64: 2534-2543, 2004

27. Kohn EC and Liotta LA: Invasion and metastasis: new approaches to an old problem. Oncology 7: 47-52, 1993.

28. Giannelli G, Bergamini C, Marinosci F, et al: Clinical role of MMP-2/TIMP-2 imbalance in hepatocellular carcinoma. Int J Cancer 97: 425-431, 2002.

29. Fan YZ, Zhang JT, Yang HC and Yang YQ: Expression of MMP-2,TIMP-2 protein and the ratio of MMP-2/TIMP-2 in gallbladder carcinoma and their significance.World J Gastroenterol 8: 1138-1143, 2002 .

30. Xiao M, Zhou NX, Huang ZQ, Lu YL, Chen LH, Wang DJ and Chang WL: The ratio of MMP-2 to TIMP-2 in hilar cholangiocarcinoma: a semi-quantitative study. Hepatobiliary Pancreat Dis Int 3: 599-602, 2004.

31. Brand K, Baker AH, Perez-Cantó A, Possling A, Sacharjat M, Geheeb $M$ and Arnold W: Treatment of colorectal liver metastases by adenoviral transfer of tissue inhibitor of metalloproteinases-2 into the liver tissue. Cancer Res 60: 5723-30, 2000 . 